\title{
Editorial
}

\section{AAI pacing for sick sinus syndrome: first choice on all counts}

The British Pacing and Electrophysiology Group guidelines for pacemaker prescription recommend single chamber atrial pacing as the most appropriate pacing mode for sinus node disease in the absence of atrioventricular conduction disturbance. ${ }^{1}$ As such it offers a rare combination of maximum clinical effectiveness for the minimum cost and a good training opportunity. However, it seems that this message has yet to be translated into pacing practice in the UK.

In this issue of Heart, Clarke et al retrospectively analyses the pacing activity for sinus node disease in a tertiary pacing centre over five years. ${ }^{2}$ They conclude that in their centre $£ 103000$ per year could have been saved by more judicious use of AAI pacing, largely by reducing the DDD implant rate. It seems likely that these potential savings are equally applicable to the rest of the UK. Current pacemaker prescribing for sinus node disease is frequently illogical, ignores current evidence, and misses out on training opportunities for junior staff.

The evidence to support atrial based pacing for sinus node disease, rather than single chamber ventricular pacing, is very strong. Numerous retrospective studies have demonstrated that mortality and morbidity are greater in ventricular only paced patients, ${ }^{3-5}$ and that the progression to chronic atrial fibrillation $(\mathrm{AF})$ is less common in patients with atrial based pacing. ${ }^{3-10}$ Although these data may be criticised for the potential biases inherent in retrospective studies, since 1994 data have been available from the first prospective randomised study. ${ }^{11}$ Andersen et al were able to demonstrate a reduction in the frequency of $\mathrm{AF}$ and incidence of thromboembolism $(5 \% \quad v 18 \%)$ with atrial pacing after three years of follow up. The most recent analysis of this study (at 5.5 years of follow up) has also shown a significant reduction in all cause mortality $(35.5 \% v 49.6 \%)$ and the degree of heart failure in patients with AAI pacing. ${ }^{12}$

Another small prospective randomised study recently compared "physiological" (AAI, DDD, DDDR or VDD) pacing with "non-physiological" (VVI or VVIR) pacing. ${ }^{13}$ This also showed a significant reduction in the incidence of chronic AF ( $8 \%$ v 22\%) and stroke $(10 \% v 22 \%)$ when patients received a physiological pacing system. To support this message, the first results of the PASE study (pacemaker selection in the elderly) show a non-significant reduction in mortality ( $6 \% v 12 \%)$, stroke $(2 \% v 4 \%)$, and all cardiac events $(10 \% v 19 \%)$ at one year of follow up. ${ }^{14}$ The authors of this study hope that further follow up will allow these trends to reach significance.

Despite this evidence to support the use of AAI pacing, the latest British Pacing and Electrophysiology Group registry data reveal that of 2700 patients paced in the UK in 1995 for sinus node disease (codes E1-E5), 44\% received ventricular only pacing systems, $45 \%$ received dual chamber devices, and only $11 \%$ received atrial only devices (UK National Pacemaker Database). This raises two important questions. Why do we implant dual chamber devices when a single chamber atrial device may provide the same benefits at a greatly reduced cost and, more importantly, why are so many patients still receiving inappropriate pacing systems?

With reference to the implantation of dual chamber devices, one explanation might be that cardiologists are afraid of the development of complete heart block in patients without a ventricular lead. However, the evidence to date suggests that with no evidence of atrioventricular (AV) nodal or His-Purkinje dysfunction at implantation, the subsequent incidence of symptomatic AV block is small. In Clarke et al's series, ${ }^{2} 5.8 \%$ of AAI paced patients developed high grade AV block over the follow up period ( $1 \%$ each year). None of these patients suffered any significant morbidity. These findings are in keeping with other retrospective studies where the incidence of $\mathrm{AV}$ block varies from $0.6-2 \%$ per annum. ${ }^{45781015-17}$ Of these, the studies reporting a high incidence include either only very small numbers or do not exclude patients at high risk. Indeed, Brandt and colleagues ${ }^{15}$ (who reported a rate of $1.8 \%$ per annum) subsequently showed that the development of AV block was predicted by pre-existing bundle branch block or bifasicular block, and the incidence of AV block in patients without these findings was much lower. Many of the other studies selected patients for AAI pacing with the additional criteria of the absence of first degree heart block and a Wenckebach point of greater than 120 beats/min. ${ }^{4516}{ }^{18-20}$ Although neither has been shown to predict progression to AV block, their use as inclusion criteria in these studies has led to their widespread acceptance in clinical practice.

In Andersen et al's prospective study, ${ }^{12}$ the incidence of $\mathrm{AV}$ block was $0.6 \%$ per annum in patients receiving AAI pacemakers. While this study excluded patients with ventricular conduction disturbance, first degree AV block, and a low Wenckebach rate, only $30 \%$ of patients evaluated were excluded on these grounds. This is in keeping with other data that suggest that the incidence of AV conduction disturbance in patients with sinus node disease is about $30 \%$. $^{21} 22$

A recent paper by Hildick-Smith et al has raised concerns that pacemaker upgrade itself may result in a high complication rate. ${ }^{23}$ This paper retrospectively reviewed the complications associated with upgrading ventricular single chamber pacemakers to DDD systems and concluded that $45 \%$ of patients experienced a "complication". However, included in the definition of complications was "a prolonged procedure" (> 102 minutes), which accounted for $42 \%$ of the complications. While this is not desirable, it probably does not constitute a major complication, it adds little to the cost of the procedure, and in this study it was not associated with other complications. Also included was "atrial lead reposition within six weeks", which accounted for a further $33 \%$ of the complications. Obviously, this cannot be applied to the upgrade of AAI pacemakers where the atrial lead is already in situ and stable. Omitting these complications would reduce the complication rate to $14 \%$. The authors also note that the complication rate was more than halved when the operator was an experienced upgrader (more than two previous procedures). Therefore, in experienced hands, upgrade of an AAI pacing system does not carry an exceptionally high complication rate.

In contrast to the perceived reasons for implanting DDD devices, there can be no logical reason for having implanted 44\% (1188) VVI pacemakers for sinus node disease in 1995. The evidence for the low incidence of AV block applies as for DDD pacing but the financial arguments against VVI pacing 
are even stronger. Sutton and Bourgeois analysed the literature on the effects of different pacing modes for sick sinus syndrome to produce a model for cost-benefit analysis. ${ }^{24}$ This model establishes the cost of various factors such as implantation, follow up, and complications (for example, AF stroke and heart failure) in arbitrary units (100 = the cost of a single chamber pulse generator). Applying this model to the UK in 1995, of 2700 patients paced for sinus node disease, $45 \%$ received dual chamber devices, $44 \%$ received ventricular only pacing systems, and $11 \%$ received atrial only devices (UK National Pacemaker Database). Using the evidence described above, it could be argued that only $30 \%$ should have received DDD systems (because of concurrent AV conduction disturbance) and $70 \%$ should have received AAI systems. Assuming the cost of single chamber pulse generator to be $£ 900$ (+VAT), the potential saving in 1995 would have been $£ 380700$ in hardware costs alone. The reduced implantation and follow up costs of AAI $v$ DDD pacing would have saved a further $£ 125478$ over the first year. Thereafter, the reduced yearly costs of follow up approximately balance the potential cost of upgrading patients who develop AV block. However, the elimination of VVI pacing for sinus node disease would have resulted in even greater savings. Essentially the cost of implanting a single chamber device is the same whether it be atrial or ventricular. Using the same model ${ }^{24}$ the health care savings from the reduction in $\mathrm{AF}$, heart failure, stroke, and subsequent disability seen with AAI pacing would amount to $£ 835797$ per annum for patients paced in 1995 (again allowing for upgrades). This is in addition to any reduction in mortality. ${ }^{12}$

Why are so many patients given inappropriate devices? It seems likely that any reasons proffered are merely excuses for ignorance or lack of interest in pacing at a senior level. Certainly, pacing appears to be predominantly a junior staff activity in many UK centres. Fear of the atrium as a site for permanent pacing leads may also be to blame. This is perhaps understandable for the inexperienced junior, as lead displacement is said to be more common in the atrium than the ventricle. ${ }^{25}$ This is only true, however, for inexperienced operators ${ }^{26}$ - the obvious solution is improved training. AAI pacing provides the ideal setting to learn atrial lead positioning as it is free from the additional complexities associated with dual chamber pacing. Combined with a greater senior input, increased use of AAI pacing should enable all junior cardiologists to become competent at placing atrial leads, even in non-specialist centres.

AAI should be the default pacing mode for sinus node disease (as recommended by BPEG) ${ }^{1}$ in the absence of AV conduction disturbance. It is almost the ideal treatmentsimple and yet elegant, it combines an excellent training opportunity with maximum clinical benefit for the lowest feasible cost.
Department of Cardiovascular Medicine,

Queen Elizabeth Hospital,

Edgbaston, Birmingham B15 2TH, UK

email: H.Y.Marshall@bham.ac.uk

1 Report of a working party of the British Pacing and Electrophysiology Group. Recommendations for pacemaker prescription for symptomatic bradycardia. Br Heart F 1991;66:185-91.

2 Clarke KW, Connelly DT, Charles RG. Single chamber atrial pacing: an underused and cost effective pacing modality in sinus node disease. Heart 1998;80:387-9.

3 Rosenqvist $M$, Brandt J, Schuller $\mathrm{H}$. Long-term pacing in sinus node disease: effects of stimulation mode on cardiovascular morbidity and mortality. Am Heart f 1988;116(1 Pt 1):16-22.

4 Santini M, Alexidou G, Ansalone G, et al. Relation of prognosis in sick sinus syndrome to age, conduction defects and modes of permanent cardiac pacsyndrome to age, conduction defects
ing. Am 7 Cardiol 1990;65:729-35.

5 Sasaki Y, Furihata A, Suyama K, et al. Comparison between ventricular inhibited pacing and physiologic pacing in sick sinus syndrome. Am f Cardiol 1991;67:771-4.

6 Feuer JM, Shandling AH, Messenger JC. Influence of cardiac pacing mode on the long-term development of atrial fibrillation. Am $\mathcal{F}$ Cardiol 1989;64: $1376-9$

7 Grimm W, Langenfeld H, Maisch B, et al. Symptoms, cardiovascular risk profile and spontaneous ECG in paced patients: a five-year follow-up study. Pacing Clin Electrophysiol 1990;13(12 Pt 2):2086-90.

8 Hesselson AB, Parsonnet V, Bernstein AD, et al. Deleterious effects of longterm single-chamber ventricular pacing in patients with sick sinus syndrome: the hidden benefits of dual-chamber pacing. $7 \mathrm{Am}$ Coll Cardiol 1992;19:1542-9.

9 Sgarbossa EB, Pinski SL, Maloney JD, et al. Chronic atrial fibrillation and stroke in paced patients with sick sinus syndrome. Relevance of clinical stroke in paced patients with sick sinus syndrome. Relevance of

10 Stangl K, Seitz K, Wirtzfeld A, et al. Differences between atrial single chamber pacing (AAI) and ventricular single chamber pacing (VVI) with respect to prognosis and antiarrhythmic effect in patients with
Pacing Clin Electrophysiol 1990;13(12 Pt 2):2080-5.

11 Andersen HR, Thuesen L, Bagger JP, et al. Prospective randomised trial of atrial versus ventricular pacing in sick-sinus syndrome [see comments]. Lancet 1994;344:1523-8.

12 Andersen HR, Nielsen JC, Thomsen PEB, et al. Long-term follow-up of patients from a randomised trial of atrial versus ventricular pacing for sick sinus syndrome. Lancet 1997;350:1210-16.

13 Mattioli AV, Castellani ET, Vivoli D, et al. Prevalence of atrial fibrillation and stroke in paced patients without prior atrial fibrillation: a prospective study. Clin Cardiol 1998;21:117-22.

14 Lamas GA, Orav EJ, Stambler BS, et al. Quality of life and clinical outcome in elderly patients treated with ventricular pacing as compared with dual-chamber pacing. N Engl f Med 1998;338:1097-104.

15 Brandt J, Anderson H, Fahraeus T, et al. Natural history of sinus node disease treated with atrial pacing in 213 patients: implications for selection of stimulation mode. 7 Am Coll Cardiol 1992;20:633-9.

16 Elshot SR, el Gamal MI, Tielen KH, et al. Incidence of atrioventricular block and chronic atrial flutter/fibrillation after implantation of atrial pacemakers; follow-up of more than ten years. Int 7 Cardiol 1993;38:303-8.

17 Kallryd A, Kruse I, Ryden L. Atrial inhibited pacing in the sick sinus node syndrome: clinical value and the demand for rate responsiveness. Pacing Clin Electrophysiol 1989;12:954-61.

18 Sasaki Y, Shimotori M, Akahane K, et al. Long-term follow-up of patients with sick sinus syndrome: a comparison of clinical aspects among unpaced, ventricular inhibited paced, and physiologically paced groups. Pacing Clin Electrophysiol 1988;11(11 Pt 1):1575-83

19 Lemke B, Holtman BJ, Selbach $\mathrm{H}$, et al. The atrial pacemaker: retrospective analysis of complications and life expectancy in patients with sinus node dysfunction. Int $\mathcal{F}$ Cardiol 1989;22:185-93.

20 Kolletis TM, Miller HC, Boon NA. Atrial pacing: who do we pace and what do we expect? Experience with 100 atrial pacemakers. Pacing Clin Electrophysiol 1990;13:625-30.

21 Carisma MB, Manalo JM, Chua WT. Atrioventricular conduction in sick sinus syndrome. Pacing Clin Electrophysiol 1988;11(11 Pt 2):1636-40.

22 Narula OS. Atrioventricular conduction defects in patients with sinus bradycardia. Analysis by His bundle recordings. Circulation 1971;44:1096110.

23 Hildick-Smith D Jr, Lowe MD, Newell SA, et al. Ventricular pacemaker upgrade: experience, complications, and recommendations. Heart 1998;79: 383-7.

24 Sutton R, Bourgeois I. Cost benefit analysis of single and dual chamber pacing for sick sinus syndrome and atrioventricular block. An economic sensitivity analysis of the literature. Eur Heart $\mathcal{F}$ 1996;17:574-82.

25 Aggarwal RK, Connelly DT, Ray SG, et al. Early complications of permanent pacemaker implantation: no difference between single and dual chamber systems. Br Heart f 1995;73:571-5.

H J MARSHALI M D GAMMAGE M J GRIFFITH
26 Parsonnet V, Bernstein A. Pacemaker implantation complication rates: an analysis of some contributing factors. F Am Coll Cardiol 1989;13:917-21. 